Seguridad Operacional y Logística Aeronáutica

Operational Safety and Aviation Logistics

Segurança Operacional e Logística Aeronáutica

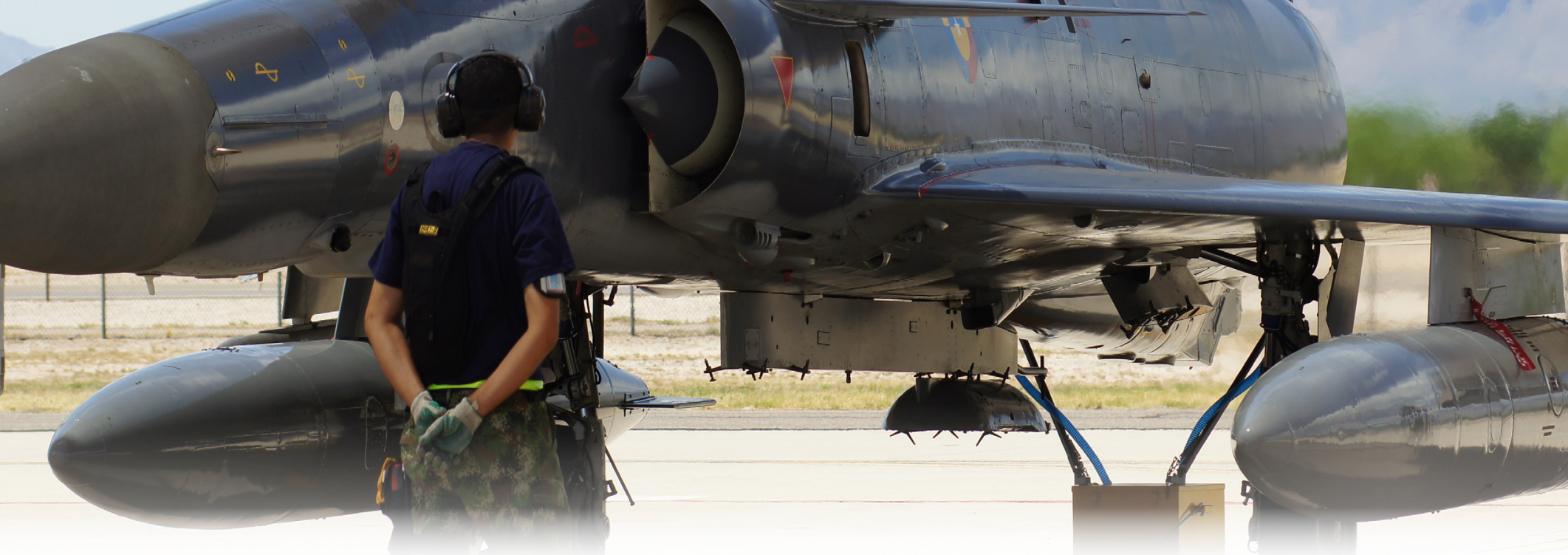

\title{
Manejo del ruido en las tripulaciones de la Fuerza Aérea Colombiana EN LAS ÚLTIMAS DOS DÉCADAS ${ }^{1}$
}

Management of Noise in Colombian Air Force Crew in the Last Two Decades²

Manejo do barulho nas tripulações da ForÇa Aérea Colombiana nas últimas duas déCADas ${ }^{3}$

Luz Caputo Silva ${ }^{4}$, María Alejandra Correa Guarín ${ }^{5}$

Fuerza Aérea Colombiana. Bogotá, Colombia.

\section{CIENCIA Y PODER AÉREO}

ISSN 1909-7050 / E- ISSN 2389-2468 / Volumen 13 / Número 1 / Enero-Junio de 2018 / Colombia / pp. 46-53 Recibido: 16/02/2016

Aprobado par evaluador: 27/02/2018

Doi: https://doi.org/10.18667/cienciaypoderaereo.585 


\section{Para citar este artículo:}

Caputo, L. y Correa, M. A. (2018). Manejo del ruido en las tripulaciones de la Fuerza Aérea Colombiana en las últimas dos décadas. Ciencia y Poder Aéreo, 13(1), 46-53. doi: https://doi.org/10.18667/cienciaypoderaereo.585.

\begin{abstract}
' Artículo de reflexión que recoge los resultados de un estudio descriptivo de los pacientes atendidos en el Centro de Medicina Aeroespacial de la Fuerza Aérea Colombiana. Este proyecto pertenece a la línea de investigación en Factores Humano de MAESO

${ }^{2}$ Reflection article that collects the results of a descriptive study of patients treated in the Centre of Air Spatial Medicine of the Colombian Air Force. This project belongs to the research line in Human Factors.
\end{abstract}

${ }^{3}$ Artigo de reflexão que coleta os resultados de um estudo descritivo dos pacientes atendidos no Centro de Medicina Aeroespacial da Força Aérea Colombiana. Este projeto pertence à linha de pesquisa de Fatores Humanos.

${ }^{4}$ Profesional Audióloga con Especialización en docencia Universitaria. Trabajó en la Fuerza Aérea Colombiana. Correo electrónico: luzacaputo@hotmail.com

${ }^{5}$ Profesional con Especialización en Otorrinolaringología con entrenamiento en Medicina Aeroespcial y capacitación en Docencia universitaria e Investigación. Otorrinolaringóloga de la Fuerza Aérea Colombiana. Bogotá, Colombia. Correo electrónico: correa10aleja@yahoo.com
Resumen: el ruido es un factor importante de contaminación auditiva en la actividad laboral, al identificarlo en la Fuerza Aérea de Colombia desde hace 20 años se inician la implementación de programas para la conservación auditiva, el objetivo de este estudio de revisión es comparar los resultados obtenidos en los diversos reportes durante este periodo para observar los cambios presentados en la población durante este tiempo con los programas implementados y su impacto en la población de tripulantes de la Fuerza Aérea de Colombia, determinando el comportamiento de la hipoacusia inducida por ruido y el uso de protectores auditivos y el autocuidado en el lugar de trabajo y fuera de él. Los resultados observado con las comparaciones y la descripción de los datos obtenidos se observa una disminución en la hipoacusia inducida por ruido, aunque está apareciendo a una edad más temprana, se encuentran otras patologías relacionadas con el vuelos y se resalta la efectividad de los programas de conservación auditiva observados en la conciencia de la población en el uso de protectores auditivos.

Palabras clave: cambios de presión, hipoacusia, prevención auditiva, protectores auditivos, ruido.

Abstract: Noise pollution is an important factor of interruption at work. Having identified it in the Colombian Air Force for 20 years, they such an institution has implemented programs for hearing conservation. The objective of this review study is to compare the results obtained in the diverse reports during this period in order to observe the changes presented in the population during this time with the implemented programs and their impact in the population of Colombian Air Force crew, determining the behavior of hearing loss induced by noise and the use of auditory protectors, as well as self-care in and out of working places. The results observed with the comparisons and the description of the obtained information show a decrease in hearing loss induced by noise. Nonetheless, although hearing loss is appearing at early age, other patologies related to the flights are found; the effectiveness of the hearing conservation programs is highlighted and observed in the daily use of auditory protection. .

Keywords: Pressure change, hearing loss, hearing conservation, ear protector, noise.

Resumo: $O$ barulho é um fator importante de contaminação auditiva na atividade laboral, ao identificá-lo na Força Aérea Colombiana há 20 anos inicia-se a implantação de programas para a conservação auditiva, o objetivo deste estudo de revisão é comparar os resultados obtidos nos vários relatórios durante este período para observar as mudanças apresentadas na população durante este período com os programas implementados e seu impacto na população de membros da tripulação da Força Aérea Colombiana, determinando o comportamento da hipoacusia induzida por barulho e o uso de protetores de audição e o autocuidado na área de trabalho e fora dele. Os resultados observados com as comparações e a descrição dos dados obtidos observa-se uma diminuição na hipoacusia induzida por barulho, embora esta aparecendo em uma idade ainda mais jovem, outras patologias relacionadas com os voos são encontradas e destaca-se a eficácia dos programas de conservação auditiva observados na consciência da população quanto ao uso de protetores auriculares.

Palavras-chave: alterações de pressão, hipoacusia, prevenção auditiva, protetores auditivos, barulho. 


\section{Introducción}

El ruido se define como toda sensación desagradable o molesta para el oído o todo sonido no deseado. Este estímulo produce alteraciones en la vía auditiva. Cuando se habla de hipoacusia inducida por ruido se hace referencia a un problema generado por la exposición a ruido de alta intensidad en forma única o continua, se puede asociar con la actividad laboral, especialmente en el área aeronáutica de la Fuerza Aérea de Colombia, debido a esto y al buscar formas de minimizar estos efectos desde hace 20 años se implementaron programas y capacitaciones referentes al cuidado auditivo en el trabajo. Se plantea a través de este artículo describir el comportamiento de hipoacusia en la población de la Fuerza Aérea durante este periodo de tiempo, a través de la descripción y comparación de los datos obtenidos de las estadísticas encontradas. De esta manera resaltar la importancia de los programas de promoción y prevención auditiva y su impacto en la población en el autocuidado auditivo y el manejo del ruido en la población y otras patologías auditivas relacionadas con el vuelo.

El ruido cuando tiene una intensidad superior a $85 \mathrm{~dB}$ es desagradable y molesta. Este estímulo produce alteraciones en la vía auditiva. Cuando se habla de hipoacusia inducida por ruido se hace referencia a un problema generado por la exposición a ruido de alta intensidad en forma única o de exposición continua, originando lesiones a nivel del oído interno en las células ciliadas, ocasionando daños como la pérdida progresiva de las células ciliadas externas por microtrauma, cambios en el metabolismo celular que lleva a apoptosis celular, pérdida de la capacidad de despolarización neuronal, alteración en la propagación del estímulo y destrucción de estructuras por macrotraumas acústicos (Hernández, 2006), generando alteraciones en la transducción del sonido y como resultado la modificación en la capacidad auditiva, alterando la comunicación.

Hay enfermedades sistémicas y hábitos que pueden influenciar la susceptibilidad individual en el origen de las lesiones que generan la hipoacusia inducida por ruido, entre ellas están: enfermedades cardiovasculares, diabetes, hipercolesterolemia, ingesta de medicamentes ototóxicos (antibióticos, diuréticos, salicilatos, quimioterapia), tabaquismo, exposición a monóxido de carbono (Sánchez y Caputo, 2000). Así mismo, se ha identificado una labilidad individual asociada a aspectos hereditarios y genético (Goyo, 2015).

Otros efectos sistémicos que produce el ruido, a partir de intensidades mayores a $50 \mathrm{~dB}$ son: trastornos del sueño, hipertensión arterial, taquicardia, taquipnea alterando el desempeño físico y sicológico (Rivera, 2013; Hernández, 2006). Puede afectar el sistema nervioso central y periférico desencadenando efectos nocivos en el comportamiento del individuo, intranquilidad, inquietud, ansiedad, depresión y rabia.

Durante la jornada laboral a ruido constante, con intensidades de $55 \mathrm{~dB}$ o más, se presentan interferencias en la comunicación, requiriendo el aumento en la intensidad conversacional, para que el lenguaje oral sea discriminado adecuadamente es necesario que su intensidad supere los $15 \mathrm{~dB}$ al ruido de fondo. De esta manera un ruido superior a 55 dB provocará dificultad en la comunicación oral, y a partir de 65 dB de ruido la conversación se torna extremadamente difícil, elevando el volumen de la fuente, creando así una mayor contaminación sonora sin lograr una comunicación adecuada (Rivera, 2013). El ruido de fondo se define como cualquier sonido que se produce en forma simultánea a otra emisión acústica, el cual puede enmascarar o alterar las comunicaciones, produciendo alteración en discriminación del lenguaje, lo que afecta la comunicación durante el desempeño laboral. Motivo por el cual la audición y su protección es fundamental en las actividades de aviación.

El ruido laboral se denomina a la contaminación acústica que se genera en el lugar de trabajo, y como hemos mencionado, afecta a los trabajadores, siendo motivo de discapacidad.

En Colombia se inicia la legislación con la aparición de la Ley 9 de 1979, con el término de "Salud Ocupacional", y se dictan las primeras medidas sanitarias a las empresas. En 1984 se disponen planes y políticas de salud modificando su objetivo desde un punto de vista curativo a uno preventivo. En 1986 la Organización Panamericana de la salud inició la utilización de la definición de medicina ocupacional o laboral, así:

Conjunto de conocimiento científicos y de técnicas destinadas a promover, proteger y mantener la salud y el bienestar de la población laboral a través de medidas dirigidas al trabajador, a las condiciones, ambiente de trabajo y a la comunidad, mediante la identificación, evaluación y control de la condiciones y factores que afectan la salud, con participación y cooperación de los trabajadores, empresarios, sectores gubernamentales y todas las instituciones involucradas. (Gomero, Zeballos, 2006; Lizarazo, Fajardo, Berrio y Quintano, 2011)

Desde 1990 a 1995 el objetivo esencial del plan de salud ocupacional nacional era reducir la ocurrencia de accidentes de trabajo y la aparición de enfermedades de origen ocupacional. Se creó el "sistema general de riesgos profesionales", donde su objetivo básico es proteger al trabajador (Lizarazo, Fajardo, Berrio y Quintano, 2011) 
En el Centro de Medicina Aeroespacial (CEMAE), desde 1995, se empezaron a desarrollar e implementar programas con mayor efectividad y cobertura al personal de vuelo, con el objetivo de prevenir enfermedades y mantener la salud auditiva, para así empezar a dar cumplimiento a estos requisitos en el campo laboral implementado por el gobierno.

Estas políticas de prevención y promoción de salud en la Fuerza Aérea, se efectuaron especialmente en el Centro de Medicina Aeroespacial, sitio de control del personal de vuelo, además, se iniciaron estudios de investigación encaminados a la caracterización de la población para enriquecer los programas de prevención y promoción del personal del vuelo. En el Encuentro de Medicina de las Fuerzas Aéreas Americanas (MEDFA) realizado en Bogotá, se presentó el trabajo de incidencia de hipoacusias en el personal de vuelo de la Fuerza Aérea de Colombia, donde se evidenció un $34 \%$ de hipoacusias inducidas por ruido en tripulantes, siendo un patrón de referencia para la aptitud psicofísica de los evaluados que tanto podrían afectar sus actividades laborales (Henao, 1993).

Con este panorama en la industria aeronáutica y el ambiente militar, en donde se identifica al ruido como un factor importante en el ambiente laboral, siendo una contaminación relevante, se reafirma la importancia de los programas de prevención y promoción auditiva, desde el ingreso a las escuelas de formación hasta la culminación de la etapa laboral, se implementan seguimientos, controles al personal para optimizar su estado de salud.

El desarrollo de este programa en la Fuerza Aérea Colombiana en los primeros 10 años se ve reflejado con el programa llevado a cabo por parte de los profesionales de fonoaudiología, los cuales se desplazaban a las unidades militares, desarrollando los siguientes puntos:

1. Capacitación mediante conferencias, materia visual y didáctica individual (afiches y folletos).

2. Elaboración de protectores auditivos a la medida con silicona INSTA-MOLD (primera silicona utilizada para protectores desde 1970).

3. Realización de audiometrías periódicas en el Centro de Medicina Aeroespacial, con entrega de protectores auditivos de espuma.

Este programa llevó a la concientización de la población de los riesgos y el cuidado que deben tener en el campo laboral. Viéndose reflejado con la utilización en masa de los protectores auditivos.

Los protectores auditivos son elementos de protección individual, cuya función es la atenuación del sonido, reduciendo los efectos del ruido. Hay diferentes tipos de protectores: de diadema, adaptados al casco y de inserción, entre ellos están de espuma, caucho y silicona (ver figura 1). (a)

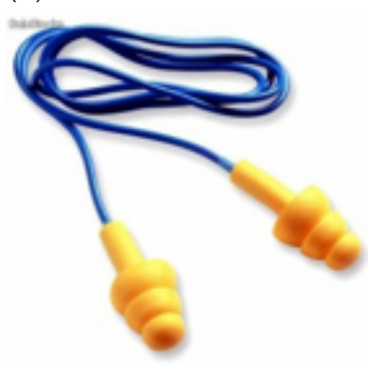

(c)

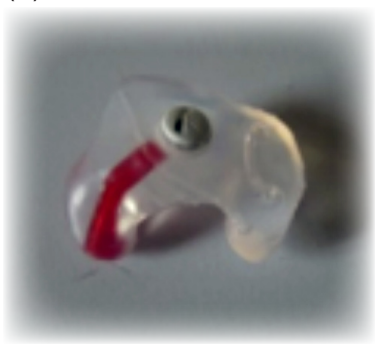

(b)

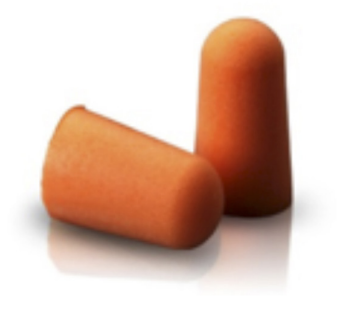

(d)

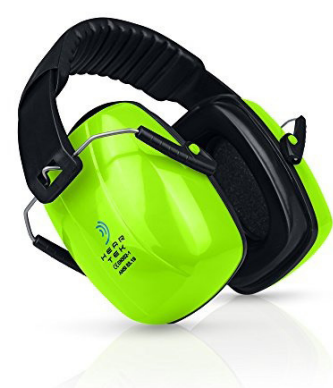

Figura 1. (a) Protectores de caucho; (b) Protectores de espuma; (c) Protectores de silicona vulcanizada; (d) diadema Fuente: recuperado de www.medop.es/contenidos/documentacion_tecnica/.../Formacion_Auditiva_Medop.pdf

El objetivo del uso de los protectores auditivos es atenuar el ruido; los protectores de inserción de espuma atenúan entre 20 a $25 \mathrm{~dB}$, los de silicona de 25 a $30 \mathrm{~dB}$ y el uso combinado de protectores de inserción y de diadema $40 \mathrm{~dB}$.

Se busca que el material del protector auditivo se adapte al conducto auditivo externo con los cambios de presión durante el vuelo. La espuma es el material con mayor adaptabilidad, con relación a los protectores de silicona el manejo de la presión durante el vuelo se ha mejorado con el uso de válvulas sónicas y la vulcanización de la silicona, mejorando la adaptabilidad de ellos.

La importancia de la limpieza y mantenimiento de los protectores auditivos, nos dan una mayor durabilidad del protector y evitan infecciones del oído externo que son dadas por la humedad en el conducto auditivo externo, generadas por el clima, descamación de la piel, alergias. Se ha descrito que los protectores de caucho son los que más generan estas alteraciones, por lo cual no es recomendable la utilización de protectores para las tripulaciones aéreas.

Se recomienda mensualmente el cambio de los protectores auditivos de espuma si su uso es diario, para los protectores anatómicos de silicona el cambio es anual, 
mientras el cambio para aquellos con material de silicona vulcanizada es de 3 a 5 años.

Según la bibliografía y con la recolección de la experiencia en los programas de conservación auditiva de la Fuerza Aérea Colombiana, se observó que los protectores auditivos de inserción de espuma eran los más maleables, limpios, menos molestos, de fácil adquisición, con menor incidencia de patología en el oído externo en la población de tripulantes (Caputo, 2004).

Con la información dada y con el objetivo de proteger al trabajador, la Fuerza Aérea Colombiana, en conjunto con salud ocupacional y seguridad aérea e industrial, crea un grupo para el manejo integral de los trabajadores con factores de riesgo laboral como el ruido, constituido por el personal médico, especialistas en salud ocupacional e ingenieros de las áreas involucradas. Uno de los resultados de este trabajo es la implementación en el ámbito militar de la protección auditiva en las actividades laborales. Viéndose reflejado en la metodología de concientización del uso de protectores auditivos, con las siguientes frases: "todo el día y todos los días" y"los protectores auditivos hacen parte del uniforme".

Según la estadística hallada en estudios en la Fuerza Aérea, se encontró una incidencia de hipoacusia en el personal de vuelo del 34 \% (Henao, 1993), posteriormente se evidenció un porcentaje del $6 \%$ de hipoacusia en el personal de vuelo, observando una disminución del $28 \%$ de la incidencia de hipoacusia en la población estudiada (Caputo, 2004, Sanchéz, Caputo, 2000). Los anteriores datos son de relevancia, en donde se refleja la evolución satisfactoria en este aspecto (ver figura 2).

\section{Metodología y resultados}

Se realizó un estudio descriptivo de los pacientes atendidos en el Centro de Medicina Aeroespacial en los últimos 10 años. Tomando como base de datos los registros estadísticos y procesándolos con el programa estadístico SPSS, se halló que se atendían un promedio anual de 1629 pacientes, entre ellos se encontró una hipoacusia inducida por ruido de un $5 \%$. Se compara con los datos obtenidos con los estudios referenciados, observando una disminución en la incidencia hipoacusia en la población.

Otro dato de relevancia encontrado es que la hipoacusia fue unilateral, con mayor afectación en el oído izquierdo. La hipoacusia aparece entre los 5 a 10 años de estar laborando, estando de acuerdo con los datos estadísticos encontrados, aunque en los últimos años este comportamiento ha tenido cambios, con una aparición más temprana de la hipoacusia en el nuevo personal militar; esto puede asociarse al uso indiscriminado de aparatos tecnológicos como celulares, iPod y otros, utilizados constantemente desde la niñez, a intensidades sonoras superiores a $85 \mathrm{~dB}$ que unidas a los primeros años de exposición a ruido laboral, revelan una posible patología auditiva precoz, esto se sustenta con base en los estudios realizados por Babish y Davis en el 2014, donde reportan un 66 \% de adultos jóvenes con alteraciones auditivas o acufenos temporales por exposición a ruido, generado en clubes nocturnos, conciertos y uso de aparatos electrónicos.

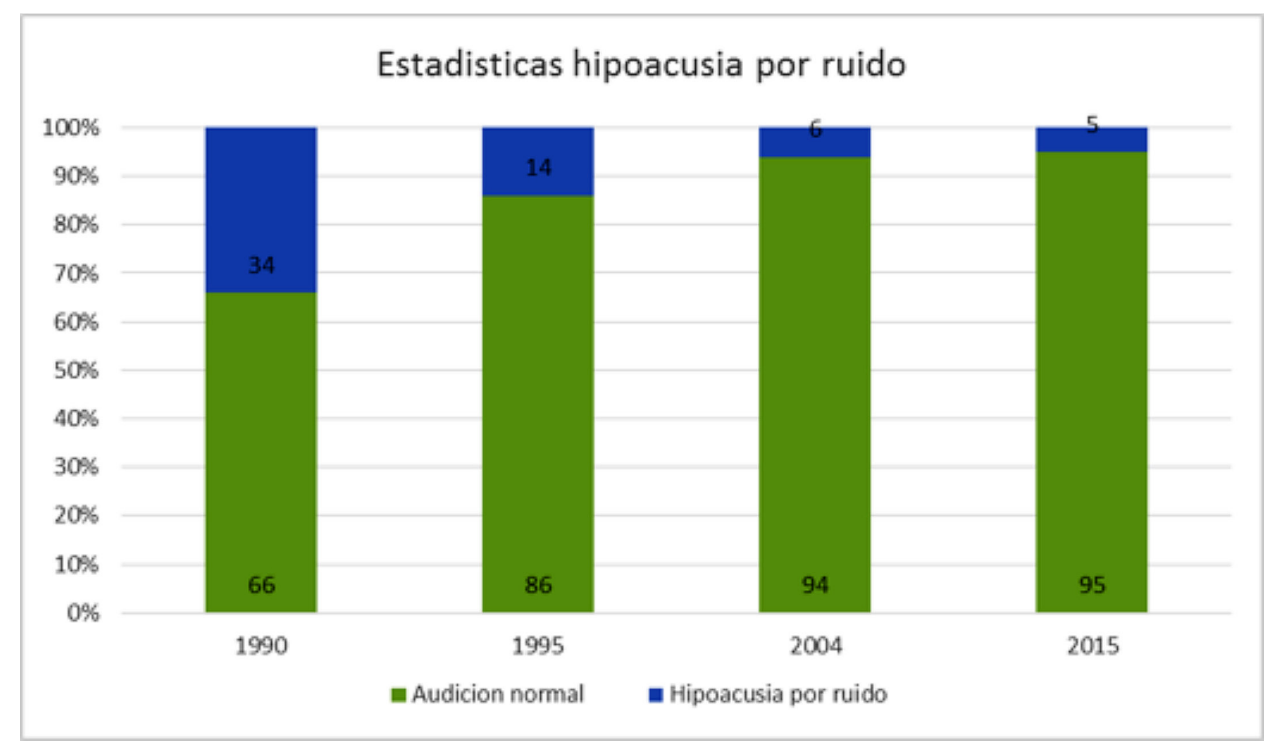

Figura 2. Comparación de hipoacusia inducida por ruido desde 1990 Fuente: elaboración propia 
Esta tendencia se corrobora con los datos obtenidos a través del personal que se presenta a las escuelas de formación, de estos un $2 \%$ entre los 17 y 26 años presentan hipoacusia en el examen, estos datos pueden explicarse con lo referido en el estudio de Babish y Davis en el 2014.

Los resultados obtenidos de los programas de prevención auditiva, además de manejar la contaminación por ruido en el lugar de trabajo, llevaron a un impacto a nivel de las actividades lúdicas del personal perteneciente a la Fuerza aérea. Los programas han continuado siendo base fundamental en la salud operacional y programas de seguridad aérea, incentivando el control audiométrico periódico, capacitaciones y el uso de protectores auditivos a través del Centro de Medicina Aeroespacial. La frase utilizada actualmente para continuar con la concientización de la conservación auditiva es: "cada uno es dueño de su propio organismo, cuídelo".

El desarrollo tecnológico en la mejoría de la protección auditiva y comunicaciones en el medio aeronáutico se evidencia con la aparición de auriculares con cancelación de ruido (Catalán, 2011). Estos auriculares pueden ser: de ruido pasivo, que bloquean el ruido de fondo para disminuir el estímulo al oído, simulan estar en una habitación tranquila para alejarse del sonido, similar a una cámara sonoamortiguada recibiendo solo el sonido que se genera de los equipo de comunicaciones o reproductores; y los auriculares de cancelación de ruido activo, los cuales requieren de una batería para accionar un pequeño micrófono exterior que recoge la muestra del ruido de fondo y produce un sonido opuesto en los auriculares para cancelar el ruido, estos no son tan efectivos como los auriculares de ruido pasivo, ya que producen un silbido al cancelar el ruido no deseado, sin embargo filtra el ruido permanente como el de un motor de avión. Las ventajas de estos auriculares con cancelación de ruido es que permiten mantener la comunicación oral, son más ligeros y pequeños en el diseño (Bose, 2015) (Rodríguez, 2013). Estos auriculares ya han sido incorporados en algunas aeronaves o son adquiridos por las tripulaciones según sus necesidades personales para su autocuidado auditivo (ver figura 3).

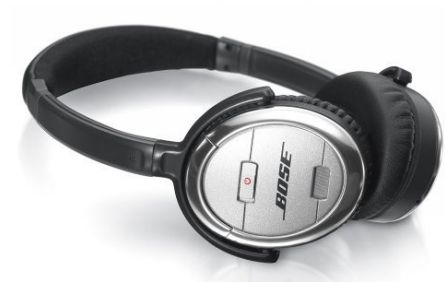

Figura 3. Auriculares de cancelación

Fuente: recuperado de www.medop.es/contenidos/documentacion_tecnica/.../Formacion_Auditiva_Medop.pdf
Otro factor que afecta el funcionamiento del sistema auditivo es el disbarismo. Definido por las alteraciones de los gases, como consecuencia de los cambios de la presión atmosférica, se produce en las cavidades del organismo, en este caso el oído. Con base en la ley de Boyle-Marriot que establece que: a temperatura constante el volumen de un gas es inversamente proporcional a la presión que se ejerce sobre dicho gas, es decir que, con el ascenso en vuelo al reducirse la presión atmosférica, el aire se expande para ocupar mayor volumen (Amezcua, 2011).

En tierra el aire del oído medio se mantiene en equilibrio con el exterior y la membrana timpánica se mantiene en su posición, durante el ascenso en vuelo la presión atmosférica va disminuyendo progresivamente y la presión que está dentro del oído es mayor, creando una diferencia de presión que empuja la membrana timpánica hacia afuera, provocando una sensación de oído tapado cuando esta presión diferencial alcanza $15 \mathrm{mmHg}$, en un ascenso de 500 a 1000 pies, la disminución de la sensación de oído tapado, se logra con la salida forzada del gas que está en el oído medio a través de la trompa de Eustaquio, con el fin de disminuir o eliminar la diferencia de presiones, regresando la membrana timpánica a la posición original. Si el ascenso continúa, este cambio se produce cada 500 a 1000 pies, de manera automática (Amezcua, 2011).

Durante el descenso se produce el efecto contrario, es decir, debido a que la presión atmosférica va aumentando la membrana timpánica se retrae por la presión diferencial creada, esta vez la presión atmosférica es mayor, para el equilibrio de las presiones en el oído el aire tiene que entrar de la rinofaringe al oído medio a través de la trompa de Eustaquio, lo cual no puede realizarse a menos que esta se abra, ya que normalmente permanece cerrada, para lograr su apertura y permitir el paso de aire se requiere de movimiento de masticación, bostezo o deglución, por medio de los cuales los músculos faríngeos llevan a la apertura de la trompa y se equilibran las presiones, este proceso no es automático sino que requiere de movimientos activos para realizarlo. En ocasiones estos movimientos no son suficientes y se requieren de maniobras para crear una presión positiva de aire en la rinofaringe, y así forzar la apertura de la trompa de Eustaquio y permitir el paso de aire al oído medio. Estas son:

- Maniobra de Válsala, en la cual se ocluye la nariz, se cierra la boca y se sopla por la nariz, provocando así una hiperpresión en las vías respiratorias que lleva a la apertura de la trompa.

- Maniobra de Toynbee, en la cual boca y nariz se cierran y se deglute, el aire del oído se aspira, lo cual permite la apertura de la trompa. 
- Maniobra de Frenzel, se deglute y oprime la lengua contra el paladar y al mismo tiempo se produce el sonido "ke", de esta manera hay una hipertensión en la nasofaringe que fuerza la apertura de la trompa.

- Maniobras de masticación y bostezo, al combinar estas dos actividades intercaladas también se logra una apertura por los movimientos de la orofaringe (Amezcua, 2011).

La trompa de Eustaquio, se encarga de equilibrar las presiones entre el oído medio y la presión atmosférica, su falla causa la alteracion de presión en el oído medio durante el vuelo.(Correa, Caputo, 2009). Se estima que los síntomas producidos por esta alteración está entre el 10-30\% del personal de vuelo y en pasajeros la incidencia es del $5 \%$ en adultos y un $25 \%$ en niños, es mayor en niños por diferencias anatómicas de la trompa de Eustaquio como: menor dimensión, menor inclinación, lo que causa dificultad para equilibrar las presiones. (Trifold, 2013).

La medida preventiva esencial para evitar cualquier tipo de complicación referente a los cambios de presión, es evitar volar con cualquier tipo de síntoma que genere inflamación de las vías respiratorias altas, alteraciones anatómicas del paladar, alteraciones en la movilidad de la membrana timpánica y obstrucciones de la trompa de Eustaquio.

Otra patología importante registrada son los barotraumas de aparición tardía, se presentan especialmente en pilotos que utilizan oxigeno durante el vuelo, se produce después de 4 a 6 horas posterior a terminar el vuelo, su fisiopatología se basa en la difusión celular del exceso de oxígeno, generando la sensación de oído tapado por aumento de la presión en el oído medio. Se recomienda en estos casos no dormir inmediatamente y realizar maniobras de trompa posterior al terminar el vuelo.

Estos eventos han contribuido a desarrollar capacitaciones tanto del personal de vuelo como del personal médico y paramédico en el manejo y prevención de esta patología (Correa y Caputo 2009) contribuyendo al desarrollo del Centro de Medicina Aeroespacial, como referente en las patologías de vuelo.

El desarrollo de la medicina ocupacional, basada en la promoción y prevención de las enfermedades de los trabajadores, en la Fuerza Aérea se evidencia a través de la implementación de programas con este objetivo, se ha observado en estos 20 años la estandarización, caracterización de la población, en riesgo con el control de la contaminación auditiva en el medio aeronáutico militar; este impacto se ve reflejado en la disminución de la incidencia de la hipoacusia evidenciada en los datos presentados; se ha disminuido en un $14 \%$ desde 1990, además se concientizó a la población del ruido en el ambiente laboral como lúdico y se estímulo el desarrollo de los programas con capacitaciones al personal médico y paramédico en este campo, acompañado del adelanto de investigaciones en este campo en la aviación militar. De igual forma, estos avances demuestran que un diagnóstico precoz y un seguimiento extenso en la población conyevan a buenos resultados El impulso en conservar la salud auditiva a las tripulaciones aéreas es vital para su desempeño laboral y para lograr efectivas operaciones aéreas. Con el objetivo de continuar en el proceso de avance en este campo, se continúa impulsando y mejorando esta iniciativa encaminada a cumplir las leyes de protección al trabajador dadas por el gobierno.

\section{Referencias}

Amezcua, L. (2011). Disbarismos. Efectos fisiológicos del vuelo. Revista virtual. España. Recuperado de http://www.semae. es/wp-content/uploads/2011/11/3.-Fisiolog\%C3\%ADa-delVuelo-Hipoxia-Disbarismos-y-Aceleraciones.pdf

Bose. (2015). Auriculares-cancelación-ruido. España. Recuperado de https://www.bose.es/content/consumer_electronics/b2 c_catalog/worldwide/websites/es_es/product/proflight_ hdst.html

Caputo, L. (2004). La audición y el ruido en la aviación. Acta de otorrinolaringología y cirugía de cabeza y cuello, 32(1)

Catalán, D. (2011). Determinación de la atenuación en dispositivos auditivos tipo orejera aplicados en la protección, comunicación y entretenimiento con control activo de ruido (tesis para optar al grado académico de licenciado en Acústica y al título profesional de ingeniero civil acústico). Chile. Recuperado de http://cybertesis.uach.cl/tesis/uach/2011/bmfcic357d/doc/ bmfcic357d.pdf

Correa, A., y Caputo, L. (2009). Otros factores de riesgo en barotrauma en los tripulantes de la Fuerza Aérea Colombiana. Bogotá.

Gomero, R., Zevallos, C. (2006). Medicina del trabajo, medicina ocupacional y del medio ambiente y salud ocupacional. Revista Hered 17(2), 105-108.

Henao, A. (1993). Ruido e hipoacusia en el personal de vuelo de la Fuerza Aérea Colombiana. Informe final XVIII comité de Medicina de las Fuerzas Aéreas Amerifcanas (MEDFAA).

Hernández, H., y Gutiérrez, M. (2006). Hipoacusia inducida por ruido: estado actual. Instituto Superior de Medicina Militar. Cuba.

Lizarazo, C. G., y Fajardo, J. M., Berrio, S. y Quintano, L. (2011). Breve historia de la salud ocupacional en Colombia. Revista Virtual Pro. Recuperado https://www.revistavirtualpro.com/biblioteca/breve-historia-de-la-salud-ocupacional-en-colombia 
Rivera, P. (2013). Efectos del ruido sobre la salud. Magazine Oír Audiología. Colombia. Recuperado de http://oiraudiologia. blogspot.com.co.

Rodríguez, Paco. (2013). Auriculares con cancelación activa del ruido. Cómo funcionan, ventajas e inconvenientes. España. Recuperado de https://www.xataka.com/audio/auricularescon-cancelacion-activa-del-ruido-como-funcionan-ventajas-e-inconvenientes

Sánchez, L., y Caputo, L. (2000). Trauma acústico y prevención auditiva en la Fuerza Aérea Colombiana. Informe final comité MEDFAA.

\section{Webgrafía consultada}

Alexandria, V. A. (2013). Medical Guidelines for Airline Passengers. Recuperado de https://www.asma.org/asma/media/asma/ Travel-Publications/paxguidelines.pdf

Centro de Información del NIDCD. (Marzo de 2014). Pérdida de audición inducida por el ruido. Recuperado de https://www. nidcd.nih.gov/es/espanol/perdida-de-audicion-inducidapor-el-ruido

Martinez, A. (29 de abril de 2008). Prehistoria [Mensaje en un blog]. Historia de la Salud Ocupacional. Recuperado de http://historiadelasaludocupacional.blogspot.com/2008/04/prehistoria.html

Royo, J. (21 de mayo de 2015). Ruido y Sociedad. [Comentatario en un foro en línea]. Recuperado de https://www.gaes.es/ viviendoelsonido/foros/post/1485/ 